\title{
Anti-Inflammatory Effect of Mallotus Philippinensis Bark Extracts in a Mouse Atopic Dermatitis Model
}

\author{
Sae Asayama, ${ }^{a, b, t}$ Ayaka Iwasaki, ${ }^{a, b, t}$ Shunya Sahara, ${ }^{c, d, t}$ Tadashi Okamoto, ${ }^{b}$ Koichi Nakaoji ${ }^{c, d}$ Kazuhiko \\ Hamad $a,{ }^{d}$ Masamitsu Ichihashi, ${ }^{b}$ and Ken-ichi Mizutani ${ }^{a^{*}}$ \\ tThese authors contributed equally. \\ ${ }^{a}$ Laboratory of Stem Cell Biology, Graduate School of Pharmaceutical Sciences, Kobe Gakuin University, Kobe, Japan. \\ ${ }^{b}$ Laboratory of Biochemistry, Faculty of Pharmaceutical Sciences, Kobe Gakuin University, Kobe, Japan.
}

'Skin Regeneration, PIAS Collaborative Research, Graduate School of Pharmaceutical Sciences, Osaka University, Suita, Japan.

${ }^{d}$ Research\& Development Division, PIAS Corporation, Kobe, Japan.

Received: April 06, 2020; Accepted: April 12, 2020; Published: April 21, 2020

*Corresponding author: Ken-ichi Mizutani, Ph.D,Specially-Appointed Professor, Laboratory of Stem Cell Biology, Graduate School of Pharmaceutical Sciences, Kobe Gakuin University, 1-1-3 Minatojima, Chuou-ku, Kobe 650-8586, Japan, Tel. No:078-974-4632; . E-mail: mizutani@pharm. kobegakuin.ac.jp

\begin{abstract}
Background: Atopic Dermatitis (AD) is a chronic inflammatory skin disease that causes functional disruption of the skin barrier. We previously found that ethanol Extracts of Mallotus Philippinensis Bark (EMPB) promoted migration of mesenchymal stem cells and improved wound healing probably through anti-inflammatory action. However, direct evidence of the anti-inflammatory effect of EMPB and the underlying mechanisms of this action remain unknown. In the present study, we evaluated whether EMPB has an effective action on anti-inflammation using an in vitro and in vivo model. We found that topical application of EMPB improved house dust miteinduced AD-like skin inflammation in NC/Nga mice. In addition, EMPB significantly inhibited various kinds of inflammatory mediators such as interleukin-1ß, inducible nitric oxide synthases, and nuclear factorkappa B in lipopolysaccharide-stimulated macrophage cells. Moreover, EMPB exhibited marked radical scavenging ability. Taken together, these results suggest that EMPB may be useful in the treatment of skin inflammatory diseases such as AD.
\end{abstract}

Keywords: Mallotus Philippinensis Bark; Anti-Inflammation; Atopic Dermatitis; Macrophages

\section{Introduction}

The Muell-Arg (Euphorbiaceae) plant Mallotus philippinensis is widely distributed and is used in traditional medicine [1]. Various parts of the plant are used for treating diabetes, and in wound healing. Recently, our group demonstrated that ethanol Extract of Mallotus Philippinensis Bark (EMPB) promoted migration of mesenchymal stem cells (MSCs), enhanced MSCs homing to the wound sites, and improved remodeling in the wound healing process in a mouse model [2]. MSCs are considered to be important during the early inflammatory phase of wound healing [3], and MSCs release cytokines that activate skin cells [4]. The inflammatory response causes recruitment and activation of many cell types and is closely regulated for the subsequent stages of proliferation to be initiated. However, little is known about the direct effect of EMPB on inflammatory response in vitro and in vivo.

Atopic dermatitis (AD) is a very common, chronic inflammatory skin disease in industrialized countries [5]. Dry skin, erythema, edema, lichenification, excoriations, oozing and crusting are key clinical manifestations of AD. AD pathogenesis has not been clearly elucidated, though skin barrier defects and altered immune responses are key components in disease development [6]. The AD onset and extension are highly influenced by genetic and environmental factors. The necessary effect of treatment is primarily reductions in skin inflammation, while minimizing side effects. Therefore, the development of natural products with multipotent anti-inflammatory action to effectively treat $\mathrm{AD}$ is important. In this study, we evaluated whether EMPB has an effective action on anti-inflammation using an in vitro and in vivo model.

\section{Materials and Methods \\ Preparation of the extract}

Mallotus philippinensis from Nepal was purchased from Maruzen Pharmaceuticals Co Ltd (Hiroshima, Japan). Chopped bark from Mallotus philippinensis was extracted for $2 \mathrm{~h}$ with aqueous ethanol $(80 \%, \mathrm{v} / \mathrm{v})$ by reflux extraction. The ethanol concentration of the isolated extract was adjusted $50 \% \mathrm{v} / \mathrm{v}$, then the extract was dried and collected as an EMPB powder.

\section{Mice}

In vivo experiments were performed in strict accordance with the recommendations in the Guide for the Care and Use of Charles River Laboratories Japan. The protocol was approved by the Committee on the Ethics of Animal Experiments of Charles River Laboratories Japan. 


\section{Induction of dermatitis}

Ten-week-old female NC/NgaTndCrlj mice were housed in a controlled room with a 12:12-hour light-dark cycle and free access to laboratory chow and water. Mice were randomly divided into the following two groups ( $\mathrm{n}=30$ for each group) at the time of initiation: vaseline-treated group (vehicle), and EMPB in vaseline-treated group (EMPB). AD-like skin lesions were induced in NC/Nga mice by topical application of BiostirAD (Biostir, Kobe, Japan), a cream-containing extract of house dust mite (Dermatophagoidesfarinae) [7]. The hair on the upper dorsal skin and the back of the ears of mice was shaved, then $4 \%$ (w/v) Sodium Dodecyl Sulfate (SDS) was applied to the shaved dorsal skin and both surfaces of each ear, and Biostir-AD was applied to the skin area twice a week for a further 3 weeks. After 1 week, $100 \mathrm{mg}$ of vaseline (vehicle) or EMPB-vaseline (10 $\mu \mathrm{g}$ EMPB) was applied to the area once a day for a further 10 days. On day 30 of the experiment, mice were sacrificed, and the dorsal skin and ear were harvested for histological analyses. Any clinical signs related to toxicity, such as loss of body weight (BW), were evaluated in all animals during an experiment period.

\section{Evaluation of the dermatitis score}

The clinical features and dermatitis scores were evaluated using the criteria that have been already reported $[8,9]$. Briefly, the development of erythema, edema, erosion, dryness, scaling, and excoriation was scored as 0 (without symptoms), 1 (mild), 2 (moderate), and 3 (severe). The sum of an individual animal's score was taken as the dermatitis score.

\section{Cell culture}

Murine macrophage RAW 264.7 cell line was used as an in vitro model to test the anti-inflammatory properties of EMPB. Cells were cultured in Eagle's Minimum Essential Medium (MEM, NacalaiTesque Inc., Kyoto, Japan) supplemented with $10 \%$ fetal bovine serum (FBS, Invitrogen, USA) in a humidified atmosphere with $95 \%$ air and $5 \% \mathrm{CO}_{2}$ at $37^{\circ} \mathrm{C}$. After being cultured in a $100 \mathrm{~mm}$ plastic dish and grown to $80 \%$ confluence, cells were detached and seeded into wells of a new plastic plate. After a $24 \mathrm{~h}$ recovery period, cells were further incubated for $24 \mathrm{~h}$ in MEM culture medium with or without EMPB at a concentration of $0.3-300 \mu \mathrm{g} / \mathrm{mL}$ in the presence or absence of $10 \mathrm{ng} / \mathrm{mL}$ lipopolysaccharide (LPS, NacalaiTesque).

\section{Real-time PCR}

Total RNA was extracted from RAW 264.7 cells using the RNeasy Mini Kit (Qiagen) according to the manufacturer's instruction. cDNA was synthesized from $1 \mu \mathrm{g}$ of RNA using QuantiTectRetrotranscriptase reaction (Qiagen). Real-time PCR (RT-PCR) was performed using SYBR green labeling (SYBR Premix Ex Taq II, Takara, Otsu, Japan) and a TP850 Real-Time PCR System (Takara), as previously described $[10,11]$. The primers used are listed in Table 1. GAPDH expression was used to normalize the samples, and each sample was run in triplicate. The relative fold change was then calculated based on the $\Delta \Delta \mathrm{Ct}$ method.

\section{Histology}

After sacrifice, the back skins and ears were fixed in $10 \%$ formalin neutral buffer solution for $24 \mathrm{~h}$, embedded in paraffin, and thin-sectioned ( $4 \mu \mathrm{m}$ thickness). The sections were obtained and stained with hematoxylin and eosine (H\&E) to monitor histological changes in the skin. To measure infiltrated mast cells, sections were stained with toluidine blue, and the numbers of mast cells were counted.

\section{Free radical scavenging activity}

The 2,2-diphenyl-1-picrylhydrazyl (DPPH) reagent, which accepts an electron or hydrogen radical to become a stable molecule, is used to detect oxidant activities. The change in DPPH absorbance after the addition of different concentrations of EMPB and $0.1 \mathrm{mg} / \mathrm{mL}$ DPPH was determined to estimate the antioxidant capacity. Vitamin C (VC) was used as a positive control.

\section{Statistics}

The dermatitis score was analyzed using a Mann-Whitney U test. The results obtained in the cell culture study were analyzed using a two-tailed Student's t-test. Data represented as mean \pm Standard Error of the Mean (SEM) in all experiments. A p value $<0.05$ was considered significant.

\section{Results}

EMPB treatment suppresses Biostir-AD-induced skin lesions in NC/Nga mice

The severity of dermatitis was evaluated for 30 days (Figure 1a). After the mite antigen was applied to the backs and ears of the mice, the clinical severity of the AD symptoms developed. Repeated application of Biostir-AD cream induced AD-like skin lesions in NC/Nga mouse. However, the EMPB treatment ameliorated the skin symptoms. The dermatitis score of back skin (Figure 1b), pinna skin (Figure 1c), and total score of both skins (Figure 1d) was determined by summating the severity index values of each mouse. In the EMPB treatment group, the dermatitis score was lower from day 21 (D21) to D30 than that in the vehicle group; especially, significance was observed at D27 (Figure 1b, 1c, 1d).

These results indicate that EMPB suppressed the spontaneously induced dermatitis in NC/Nga mice (Figure 1e). During the experiment, changes in BW were measured to assess the general health status of mice (Figure 1f), and we confirmed that application of EMPB did not significantly affect the BW compared with the control group. 
a

Scoring of dermatitis

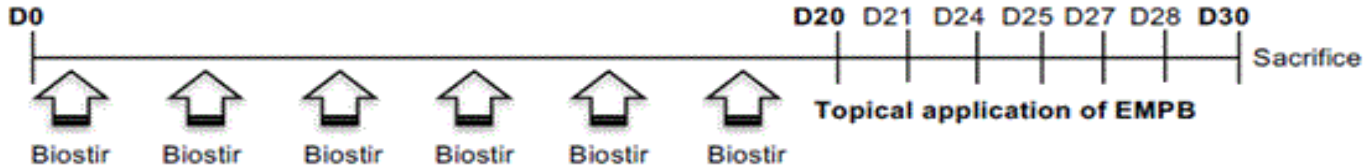

b Back skin

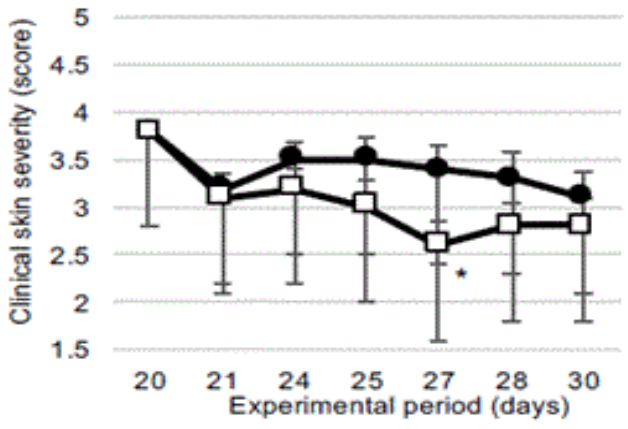

C Pinna skin

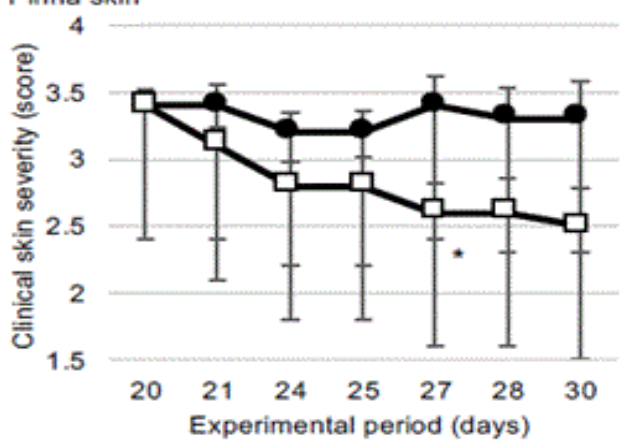

d Total (Back skin + Pinna skin)

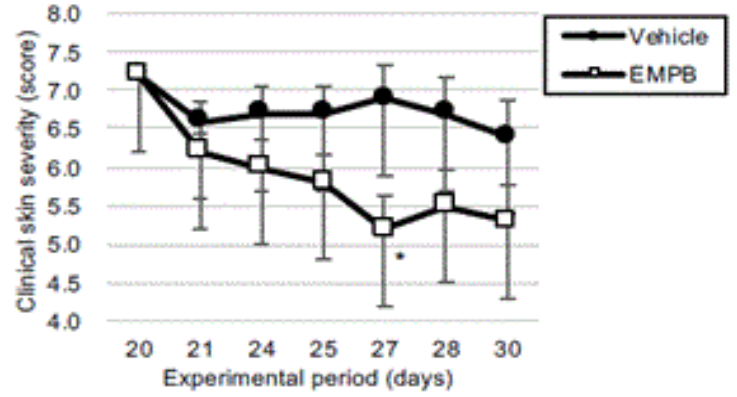

e
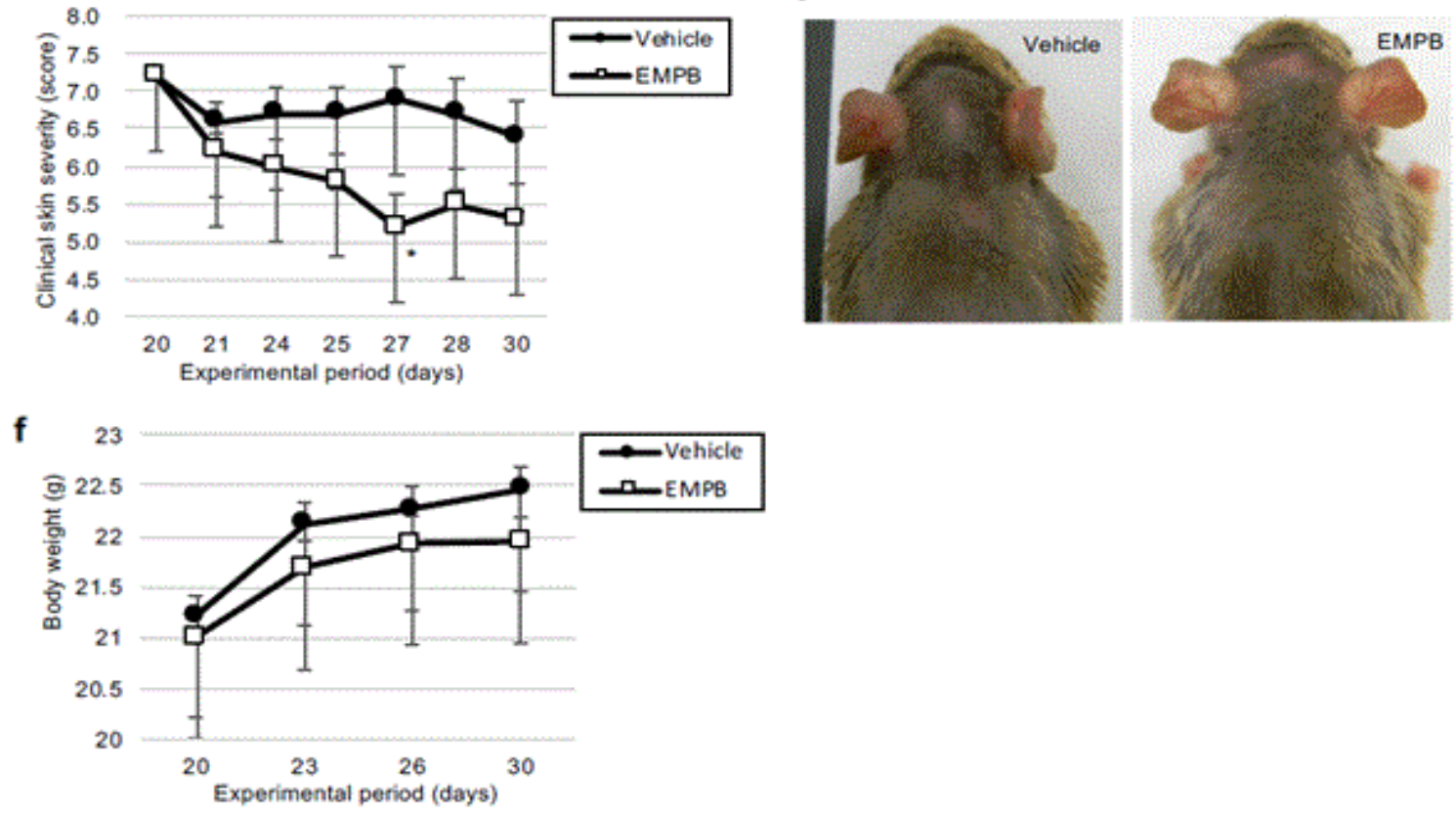

Figure 1: Effects of EPMB treatment on the development of atopic dermatitis-like skin lesions in NC/Nga mice. (a) The experimental scheme for induction of house dust mite-induced atopic dermatitis-like skin inflammation and EMPB treatment. Clinical severity scores of mice were measured on day (D)20, 21, 24, 25, 27, 28, and 30 of the experimental period in the (b) back skin and (c) pinna skin. (d) Total score of both back skin and pinna skin. (e) House dust mite-induced skin lesions. Photographs were taken on the last day (D30) of the experimental period. (f) The changes in body weight (BW) of mice. Data represent mean \pm SEM of all experiments. *p $<0.05$.

Citation: Ken-ichi Mizutani, Sae Asayama, Ayaka Iwasaki, Shunya Sahara et al.(2020) Anti-Inflammatory Effect of Mallotus Philippinensis Bark Extracts in a Mouse Atopic Dermatitis Model. Clin Res Dermatol Open Access 7(1): 1-5. DOI: 10.15226/2378- 


\section{EMPB improves Biostir-AD-induced histopathological features in NC/Nga mice}

Specimens from the back skin and ears of NC/Nga mice were examined histopathologically. As shown in Figure 2, H\&E staining revealed AD-like symptoms, such as epidermal thickening (Figure $2 \mathrm{a}, \mathrm{b}$ ) and infiltration into the dermis (Figure 2c). However, EMPB treatment significantly reduced epidermal hyperplasia (Figure $2 \mathrm{a}, \mathrm{b}$ ) and immune cell infiltration (Figure 2c), suggesting that EMPB suppressed the Biostir-AD-induced inflammation. To investigate this phenomenon further, we measured the effect of EMPB on the infiltration of mast cells by staining with toluidine blue. In the EMPB treatment group, the number of the mast cells was decreased compared with the control group (Figure $2 \mathrm{~d}, \mathrm{e}$ ).

Effect of EMPB on expression of pro-inflammatory cytokines in macrophage cells

In RAW264.7 macrophage cells, $10 \mathrm{ng} / \mathrm{mL}$ LPS stimulation increased the expression of pro-inflammatory cytokines such as interleukin (IL)-1ß (Figure 3a). To observe the effect of EMPB on cytokine expression in RAW 264.7 cells, the cells were treated with EMPB $(0.3-300 \mu \mathrm{g} / \mathrm{mL})$ in the presence of LPS (Figure 3b). EMPB reduced the IL-1ß mRNA expression level in a dose-dependent manner from 0.3 to $300 \mu \mathrm{g} / \mathrm{mL}$. Also, we determined the potential anti-inflammatory properties of EMPB by determining the mRNA expression levels of various kinds of inflammatory mediators at both 30 (Figure 3c) and $300 \mu \mathrm{g} / \mathrm{mL}$ (Figure 3d). Among proinflammatory mediators, EMPB treatment significantly reduced the expression of IL-1ß, inducible nitric oxide synthases (iNOS), and cyclooxygenase (COX)-2 compared with the LPS-stimulated macrophage control cells. These results suggest that EMPB has beneficial effects in LPS-stimulated macrophages, supporting that EMPB may contain some useful anti-inflammatory components.

Effect of EMPB on NF- $\mathrm{B}$ activation and its free radical scavenging activity

Nuclear Factor-Kappa B (NF- $\mathrm{B}$ ) is a master switch of inflammatory gene expression that regulates the levels of IL-1ß and iNOS. Therefore, we investigated the effect of EMPB on the LPS-induced activation of NF- $\mathrm{BB}$ (Figure 4a). RT-PCR analysis showed that the mRNA expression levels of NF- $\kappa B$ component factors, such as p65, p50, and p52, were significantly suppressed in EMPB-treated cells compared with control cells.

$\mathrm{AD}$ patients were more prone to damage caused by reactive oxygen species (ROS) or oxidants [12]. Also, it has been shown that crosstalk between ROS levels and NF- $\mathrm{\kappa B}$ signaling is involved in pathological conditions [13]. In this study, we confirmed that EMPB exhibited marked radical scavenging ability and scavenged $71.7 \pm 12.1 \%$ of the DPPH free radical; the positive control (vitamin C) scavenged $91.8 \pm 0.9 \%$. These results suggest that EMPB is effective for preventing $\mathrm{AD}$ by inhibiting the effect of inflammation, which is partly regulated by NF- $\kappa \mathrm{B}$ activation and cellular ROS levels (Figure 4c).

\section{Discussion}

In the present study, we investigated the effects of EMPB on house dust mite-induced AD-like skin lesions in NC/Nga mice, an animal model of human $\mathrm{AD}[14,15]$. Inflammatory responses play important roles in normal and pathological healing of diseases such as AD. Following pathologic onset, various factors activate the immune system, leading to a local inflammatory response [16]. Pro-inflammatory cytokine production, such as IL-6, IL-1 $\beta$, and Tumor Necrosis Factor (TNF), and subsequent stimulation of oxygen-centered free radical production is induced. In addition, NF- $\kappa \mathrm{B}$ constitutes a family of transcriptional regulator essential for inducible expression of many cellular signaling pathways, including iNOS, which is involved in cell survival and the inflammatory pathway [17]. Furthermore, inflammatory mediators such as IL-1 $\beta$, and nitric oxide (NO) are produced by activated macrophages, which lead to fatal damage in inflammatory diseases. The high iNOS activity typically occurs in an oxidative environment, and high levels of NO induced by proinflammatory cytokines interact with superoxide anions leading to cell toxicity [18].

In this study, we used LPS-stimulated macrophages as an in vitro model of inflammation. The EMPB treatment attenuated LPS-induced inflammation. This study found that IL-1ß, iNOS, COX-2, and NF- $\kappa$ B levels increased significantly in LPStreated cells, whereas levels were decreased by treatment with EMPB. This supports that EMPB protects the cell and prevents inflammatory symptoms by decreasing the mRNA expressions of IL-1ß, iNOS, COX-2, and NF- $\kappa$ B(Figure 4c).

In our preliminary screening experiment, EMPB was found to be activate MSCs migration and improved wound healing probably through anti-inflammatory action. MSCs may play an important role in tissue regeneration $[19,20]$. MSC also showed extensive immunomodulatory effects such as the secretion of various cytokines $[20,21,22]$. Previously, other group reported that EMPB components such as cinnamtannin B-1 exhibit antioxidant properties [23], antimicrobial activities [24], antiplatelet aggregation [25], and promotion of the migration of adipose tissue-derived stromal cells in vitro[26]. Therefore, the anti-inflammatory effect of EMPB in a mouse AD model may also be attributed to the induction of active MSCs.

In conclusion, we found that EMPB inhibited inflammatory responses induced by pro-inflammatory cytokines and NF- $\mathrm{BB}$ in LPS-stimulated macrophage cells. We also demonstrated that EMPB can ameliorate AD-like skin inflammation in a mouse model of AD in NC/Nga mice. These results may support the novel application of EMPB for treatment of AD and other inflammatory skin diseases. 

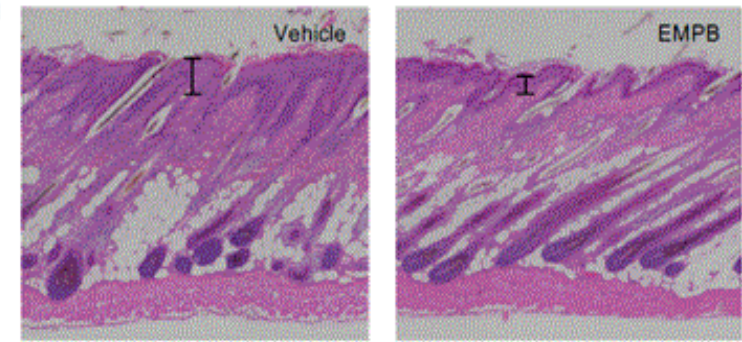

C

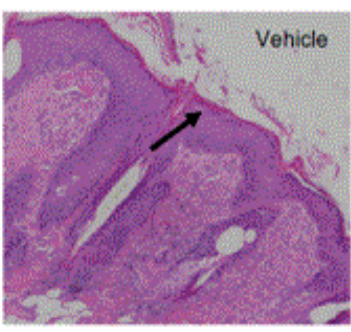

d
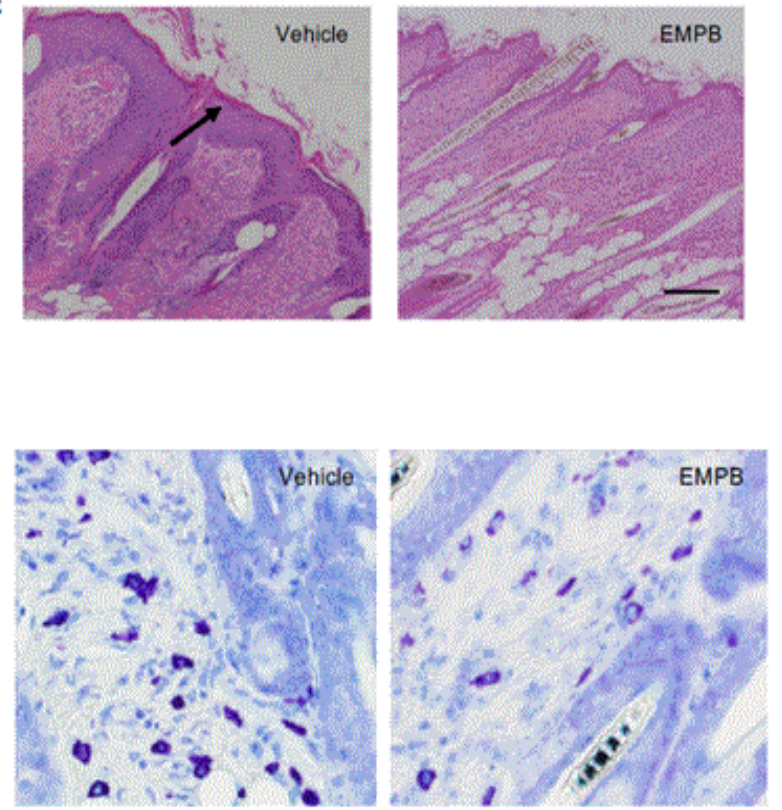

b

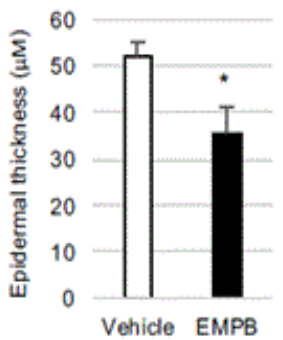

e

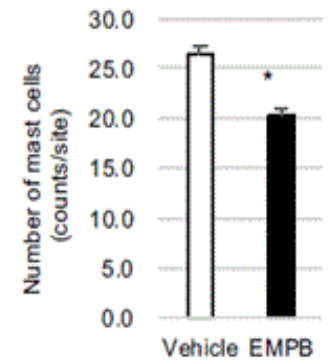

Figure 2: Effects of EMPB treatment on histological features and infiltration of mast cells into the skins of NC/Nga mice. The sections from NC/Nga mice were stained with hematoxylin and eosin-stained dorsal skin lesions. (a, b) EMPB treatment reduced epidermal thickness. Data are representative of two independent experiments and presented as mean \pm SEM of $n=30$ mice per group. (c) EMPB treatment reduced cellular infiltration into the dermis. $(\mathrm{d}, \mathrm{e})$ The number of mast cells stained by toluidine blue is reduced in the EMPB treatment group. Data represent mean $\pm \mathrm{SEM}$ of all experiments. ${ }^{*} \mathrm{p}<0.05$. 


\begin{tabular}{|c|c|c|c|}
\hline Table 1: Primer Sequences used in real-time PCR. & \multicolumn{1}{|c|}{ Forward Primer } & Reverse Primer \\
\hline Gene & Species & AGCTTGTCATCAACGGGAAG & TTTGATGTTAGTGGGGTCTCG \\
\hline GAPDH & mouse & CAGGCAGGCAGTATCACTCA & TGTCCTCATCCTGGAAGGTC \\
\hline IL-1ß & mouse & GTGGTGACAAGCACATTTGG & AAGGCCAAACACAGCATACC \\
\hline iNOS & mouse & AGAAGGAAATGGCTGCAGAA & CCCCAAAGATAGCATCTGGA \\
\hline COX-2 & mouse & TCAATGGCTACACAGGACCA & GGCAGAGGTCAGCCTCATAG \\
\hline p65 & mouse & CACCTAGCTGCCAAAGAAGG & GCAGGCTATTGCTCATCACA \\
\hline p50 & mouse & TCATGTGGCAGAGTTTCAGC & CCACCTCCTTCTTCTGCTTG \\
\hline
\end{tabular}

a

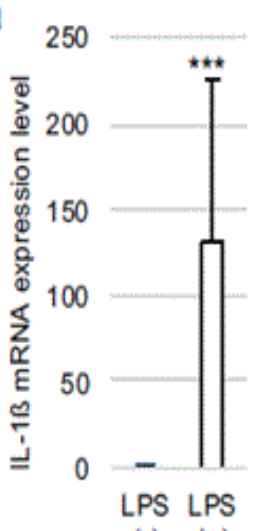

$(-) \quad(+)$

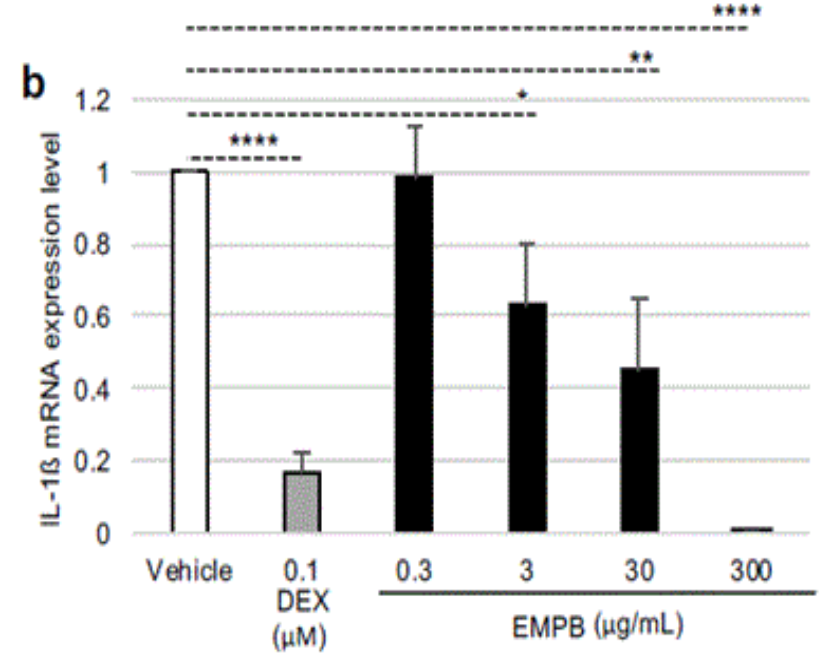

C

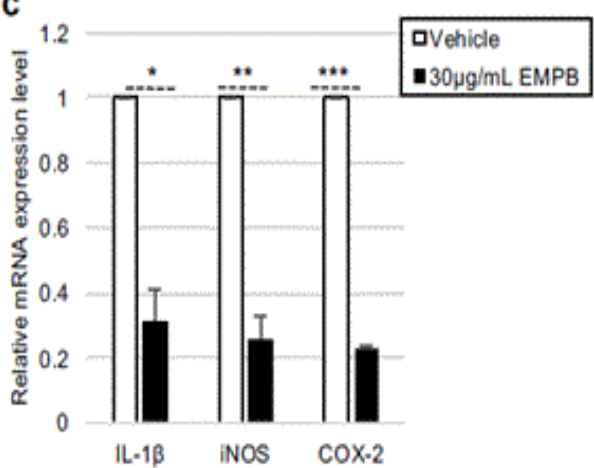

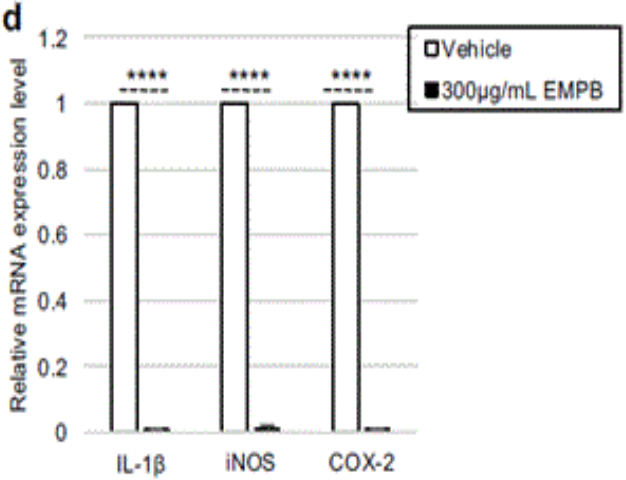

Figure 3: Effects of EMPB on pro-inflammatory mediators in the LPS-stimulated RAW264.7 cells. (a) LPS stimulation increased IL-1ß mRNA expression levels in RAW264.7 macrophage cells. (b) Cells were treated with various concentrations of EMPB ranging from 0.3 to $300 \mu \mathrm{g} / \mathrm{mL}$ in the presence of LPS. Dexamethazone (DEX) is the positive control of anti-inflammatory effect assay with a concentration of $0.1 \mu \mathrm{M}$. RT-PCR analysis showing mRNA expression of pro-inflammatory mediators in the LPS-stimulated cells with (c) 30 or (d) $300 \mu \mathrm{g} / \mathrm{mL}$ EMPB. Data represent mean \pm SEM of all experiments. ${ }^{*} \mathrm{p}<0.05,{ }^{* *} \mathrm{p}<0.01,{ }^{* * *} \mathrm{p}<0.001,{ }^{* * * *} \mathrm{p}<0.0001$ 
a

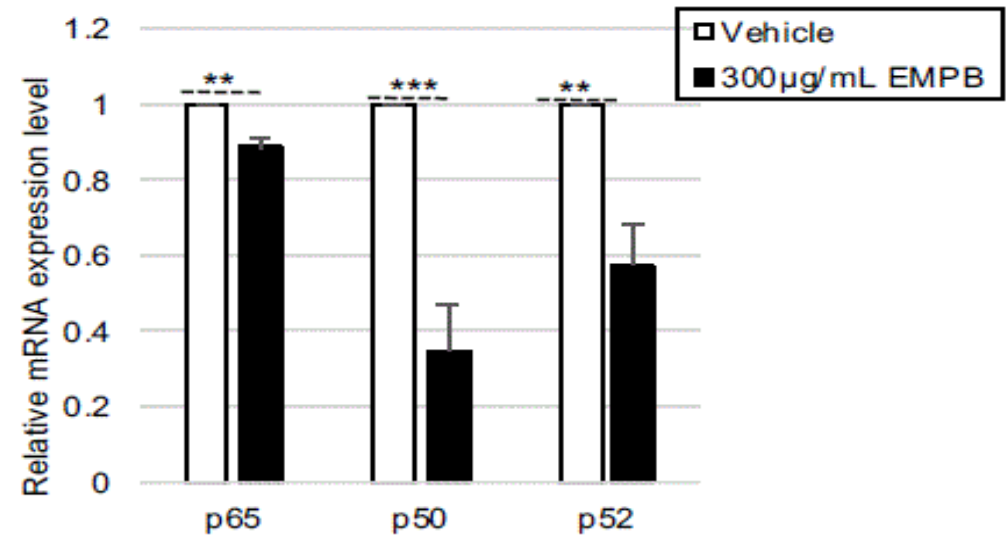

b 1.2

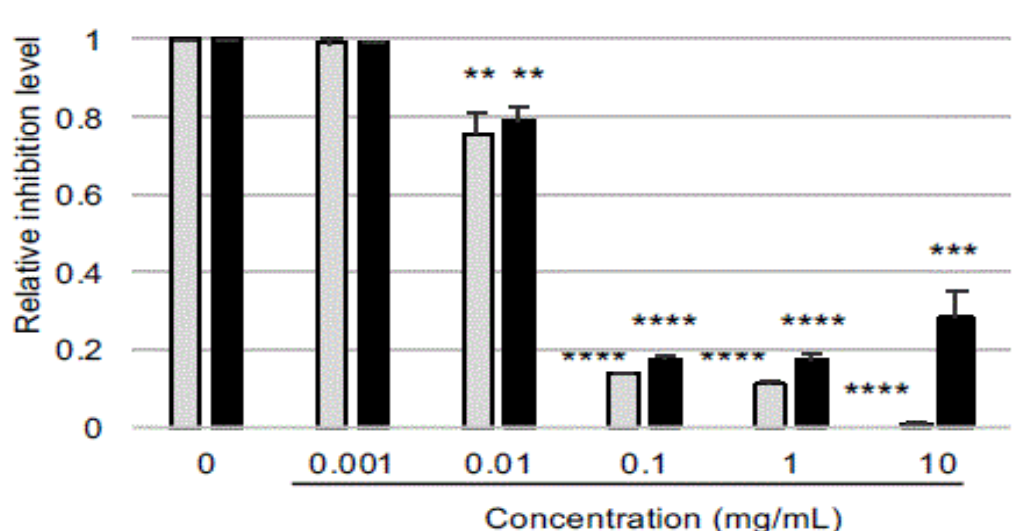

Concentration $(\mathrm{mg} / \mathrm{mL})$

$\mathbf{C}$

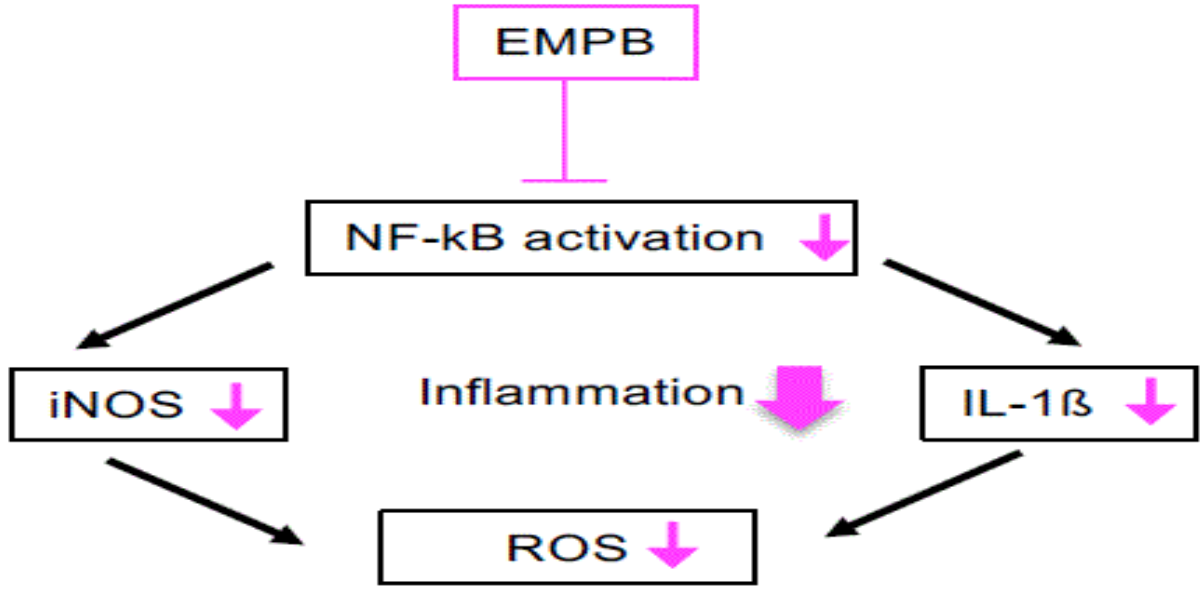

Figure 4: Effects of EMPB on the LPS-induced activation of NF-кB and ROS levels. (a) The mRNA expression levels of p65, p50, and p52 were significantly inhibited by the treatment of $300 \mu \mathrm{g} / \mathrm{mL}$ EMPB in the LPS-stimulated RAW264.7 cells. (b) The effect of anti-oxidative activity assays on EMPB at different concentrations. Vitamin C (VC) is the positive control of DPPH radical scavenging capacity assay. Data represent mean \pm SEM of all experiments. ${ }^{* *} \mathrm{p}<0.01,{ }^{* * *} \mathrm{p}<0.001,{ }^{* * * *} \mathrm{p}<0.0001$. (c) Schematic illustration of anti-inflammation mechanisms of EMPB. 


\section{Acknowledgement}

The authors thank Mrs. Yoshimi Abe for the excellent technical assistance

\section{References}

1. J Sharma, RA Varma. Review on Endangered plant of Mallotus philippinensis (Lam) M. Arg. Pharmacologyonline. 2011;3:12561265.

2. Furumoto T, Ozawa N, Inami Y, Toyoshima M, Fujita K, Zaiki K, et al. Mallotus philippinensis bark extracts promote preferential migration of mesenchymal stem cells and improve wound healing in mice. Phytomedicine. 2014;21(3):247-253.

3. Lau K, Paus R, Tiede S, Day P, Bayat A. Exploring the role of stem cells in cutaneous wound healing. Exp Dermatol. 2009;18(11):921-933.

4. Wu Y, Zhao RC, Tredget EE. Concise review: bone marrow-derived stem/progenitor cells in cutaneous repair and regeneration. Stem Cells. 2010;28(5):905-915.

5. Flohr C, Mann J. New insights into the epidemiology of childhood atopic dermatitis. Allergy. 2014;69(1):3-16.

6. Boguniewicz M, Leung DY. Atopic dermatitis: a disease of altered skin barrier and immune dysregulation. Immunol Rev. 2011;242(1):233246.

7. Yamamoto M, Haruna T, Yasui K, Takahashi H, Iduhara M, Takaki S, et al. A novel atopic dermatitis model induced by topical application with dermatophagoides farinae extract in NC/Nga mice. Allergol Int. 2007;56(2):139-148.

8. Yamaguchi T, Maekawa T, Nishikawa Y, Nojima H, Kaneko M, Kawakita $\mathrm{T}$, et al. Characterization of itch-associated responses of NC mice with mite-induced chronic dermatitis. J Dermatol Sci. 2001;25(1):20-28.

9. Matsuoka H, Maki N, Yoshida S, Arai M, Wang J, Oikawa Y, et al. A mouse model of the atopic eczema/dermatitis syndrome by repeated application of a crude extract of house-dust mite Dermatophagoides farinae. Allergy. 2003;58(2):139-145.

10. Komabayashi SM, Yamanishi E, Watanabe C, Okamura M, Tabata H, Iwai R, et al. Spatiotemporally dependent vascularization is differently utilized among neural progenitor subtypes during neocortical development. Cell Reports. 2019;29(5):1113-1129.

11. Inoue $M$, Iwai $R$, Tabata $H$, Konno D, Suzuki-Komabayashi M, Watanabe $\mathrm{C}$, et al. Prdm16 is crucial for progression of the multipolar phase during neural differentiation of the developing neocortex. Development. 2017;144(3):385-399.

12. Sivaranjani N, Rao SV, Rajeev G. Role of reactive oxygen species and antioxidants in atopic dermatitis. J Clin Diagn Res. 2013;7(12):26832685.

13. Morgan MJ, Liu ZG. Crosstalk of reactive oxygen species and NF- $\kappa B$ signaling. Cell Res. 2011;21(1):103-115.

14. Matsuda H, Watanabe N, Geba GP, Sperl J, Tsudzuki M, Hiroi J, et al. Development of atopic dermatitis-like skin lesion with IgE hyperproduction in NC/Nga mice. Int Immunol. 1997;9(3):461-466.

15. Vestergaard C, Yoneyama H, Murai M, Nakamura K, Tamaki K, Terashima $\mathrm{Y}$, et al. Overproduction of Th2-specific chemokines in $\mathrm{NC} /$ Nga mice exhibiting atopic dermatitis-like lesions. J Clin Invest.
1999;104(8):1097-1105.

16. Koh TJ, Pietro LA. Inflammation and wound healing: the role of the macrophage. Expert Rev Mol Med. 2011;13:e23

17. Perkins ND. Integrating cell-signalling pathways with NF-kappaB and IKK function. Nat Rev Mol Cell Biol. 2007;8(1):49-62.

18. Huang SH, Wu SH, Lee SS, Chang KP, Chai CY, Yeh JL, et al. Fat Grafting in Burn Scar Alleviates Neuropathic Pain via Anti-Inflammation Effect in Scar and Spinal Cord. PLoS One. 2015;10(9):e0137563.

19. Phinney DG, Prockop DJ. Concise review: Mesenchymal stem/ multipotent stromal cells: The state of transdifferentiation and modes of tissue repair-Current views. Stem Cells. 2007;25(11):2896-2902.

20. Uccelli A, Moretta L, Pistoia V. Mesenchymal stem cells in health and disease. Nat Rev Immunol. 2008;8(9):726-736.

21. Fiorina P, Voltarelli J, Zavazava N. Immunological applications of stem cells in type 1 diabetes. Endocr Rev. 2011;32(6):725-754.

22. Cho BS, Kim JO, Ha DH, Yi YW. Exosomes derived from human adipose tissue-derived mesenchymal stem cells alleviate atopic dermatitis. Stem Cell Res Ther. 2018;9(1):187.

23. Lopez JJ, Jardin I, Salido GM, Rosado JA. Cinnamtannin B-1 as an antioxidant and platelet aggregation inhibitor. Life Sci. 2008;82(1920):977-982.

24. Bouaziz A, Salido S, Palomino PJ, Sanchez A, Altarejos J, Bartegi A, et al. Cinnamtannin B-1 from bay wood reduces abnormal intracellular $\mathrm{Ca}^{2+}$ homeostasis and platelet hyperaggregability in type 2 diabetes mellitus patients. Arch. Biochem Biophys. 2007;457(2):235-242.

25. Idowu TO, Ogundaini AO, Salau AO, Obuotor EM, Bezabih M, Abegaz BM. Doubly linked, A-type proanthocyanidin turner and other constituents of Ixora coccinea leaves and their antioxidant and antibacterial properties. Phytochemistry. 2010;71(17-18):20922098.

26. Wang H, Liu TQ, Guan S, Zhu YX, Cui ZF. Protocatechuic acid from Alpinia oxyphylla promotes migration of human adipose tissuederived stromal cells in vitro. Eur J Pharmacol. 2008;599(1-3):24-31. 